\title{
Laboratorio Nacional de Nanotecnología LANOTEC y PYMES hacia procesos eficientes de producción de biocombustibles: I+D+i de la mano de la nanotecnología en Costa Rica
}

\author{
Federico Solera Jiménez*, José Roberto Vega Baudrit**
}

\section{Situación energética e innovación en Costa Rica}

El Plan Nacional de Ciencia, Tecnología e Innovación 2011-2014 identifica siete áreas programáticas de intervención estratégica: Ciencias de la Tierra y el Espacio, Nanotecnología y Nuevos Materiales, Biotecnología, Capital Natural, Salud: Enfermedades Emergentes, Energías Alternativas y Tecnologías Digitales (Ministerio de Ciencia y Tecnología 2011).

En Costa Rica, respecto al área de energías alternativas, el abastecimiento y uso sostenible de la energía representan un problema estratégico para los objetivos de desarrollo del país. Sin embargo, el tema de energías muestra una gran contradicción: a pesar del gran potencial del país para la generación de energías verdes, la mayor proporción de la energía que este consume está basada en combustibles fósiles. Así, el sector que presenta mayor dependencia de combustibles fósiles (petróleo) es el de transporte. Existe conciencia nacional sobre la necesidad de desarrollar iniciativas que complementen la directriz en política energética que propone la actual administración, que se basa principalmente en la carbono neutralidad y en la sostenibilidad y eficiencia en el uso de la energía (IV Plan Nacional de Energía 20122030).

La necesidad más urgente se centra en disminuir la dependencia de los combustibles fósiles, en especial del petróleo, y mejorar la eficiencia energética por medio de las energías limpias. Como país que depende de la importación de combustibles fósiles, aun teniendo la capacidad de producir energía limpia, es fundamental desencadenar acciones que exploten dicha capacidad y provoquen un cambio en la matriz energética.

Es urgente que Costa Rica inicie un plan de sustitución del consumo de combustibles fósiles en el sector transporte, el más vulnerable de esa dependencia. Como ejemplos exitosos en el mundo en general, países como Portugal, Alemania e Islandia, entre otros, han logrado cambios sustantivos en el corto plazo. Costa Rica se ha propuesto la neutralidad en emisiones de carbono y el logro de esta meta está directamente relacionado con los cambios que se hagan en la matriz energética.

\footnotetext{
* Investigador Asociado. Laboratorio Nacional de Nanotecnología. Dirección Postal: 1174-1200 Pavas. San José. Correo electrónico: fsolera@cenat.ac.cr Fax: (506) 22320423.

** Director General. Laboratorio Nacional de Nanotecnología. Correo electrónico: jvegab@cenat.ac.cr.
} 
Tres cuartas partes del consumo energético proviene de hidrocarburos: un problema de raíces macroeconómicas más que de la mera dependencia energética del país. (IV Plan Nacional de Energía 2012-2030).

Como iniciativa importante para el país sobresale la Política Energética Nacional 2010-2014, basada en producir energía limpia en forma amigable con el ambiente, disminuir la dependencia del petróleo, sustituir los combustibles fósiles, contar con un sistema eficiente de transporte, hacer más competitivas las instituciones relacionadas con el sector y promover el uso de tecnología digital para evitar el desplazamiento de las personas.

Sobresale también el VI Plan Nacional de Energía 2012-2030 de Costa Rica, presentado por la Dirección Sectorial de Energía del Ministerio de Ambiente, Energía y Telecomunicaciones, donde se puede concluir que las expectativas de crecimiento económico nacional, con tasas promedio del PIB del 5\% anual en los próximos 15 años, hacen prever incrementos importantes en el consumo de energía, que dejan corto la oferta actual. Por ejemplo, sólo para el año 2016, la demanda de energía se duplicará, lo que obliga a actuar de modo que el crecimiento del sector se planifique correctamente, haciendo un esfuerzo por lograr la mayor eficiencia posible tanto en la oferta como en el consumo de la energía.

Así, el Plan Nacional de Energía plantea estrategias en las áreas de desarrollo de potencial energético, producción e importación, almacenamiento, transporte y distribución, administración de la demanda, financiamiento y política de precios, el área institucional y organización y conservación de recursos y ambiente. La política energética se propone lograr una oferta energética de calidad y con precios competitivos, con participación pública y privada en el marco de regulaciones claras y transparentes, que respete y promueva el proceso de gestión integrada de cuencas, con participación creciente y sostenida de fuentes nuevas y renovables y menos contaminantes, con prevención y mitigación de las afectaciones ambientales, con menor vulnerabilidad y riesgos en todas las etapas de la cadena. Por otro lado, busca racionalidad y eficiencia en el uso de la energía, con empleo de tecnologías que permitan el aprovechamiento de fuentes alternas y equipamiento de mayor eficiencia, confiable y ambientalmente amigable, contando para ello con una cultura de uso racional en todos los sectores de consumo.

Cabe destacar también el Programa Nacional de Biocombustibles propuesto en 2008, que, en su rubro de Investigación y Desarrollo de la Bioenergética Nacional, busca impulsar el fortalecimiento del conocimiento científicotecnológico local para apoyar la producción y uso de biocombustibles, proponiendo la creación de un sistema de financiamiento para la investigación de la bioenergética nacional, para cubrir las necesidades de I+D, y la generación de experiencias en proyectos de biocombustibles. Esto basado en un modelo que plantea trabajar en tres ejes: sostenibilidad ambiental de los factores de producción, eficiencia productiva y nuevas variedades.

Pese a este panorama nacional, aún son escasas las iniciativas tanto públicas como privadas para incursionar tanto en la producción de biocombustibles, como en la I+D+i que optimice energética y ambientalmente la producción, para la generación de ventajas competitivas que consistirían en nuevas fuentes de materias primas, procesos tecnológicos eficientes para su aprovechamiento, generando productos de alta calidad, que cubran la creciente demanda energética nacional, e inclusive el país podría exportar estas eventuales nuevas tecnologías. 


\section{LANOTEC EN ALIANZA CON PYMES COMO INICIATIVA}

\section{HACIA UNA PRODUCCIÓN EFICIENTE DE BIOCOMBUSTIBLES}

Desde hace unos diez años, Costa Rica ha venido sumándose paulatinamente a la tendencia mundial de acercamiento cada vez mayor entre el sector productivo (desde las corporaciones multinacionales hasta la pequeña y micro empresa), el sector gubernamental, y el sector académico, éste como impulsor de la investigación y conocimiento. Una mayor y más profunda colaboración entre estos sectores implica un mejor y mayor desarrollo del país.

LANOTEC es un espacio de investigación interdisciplinario cuya misión es el desarrollo científico y tecnológico a través de la aplicación de nanociencia y nanotecnología a la resolución de problemas, así como la transferencia de conocimientos desde el espacio académico hacia la industria

Bioenergy Solutions of Central America S. A. (BSCA) es un emprendimiento (PYME) fundado en 2010 cuya creación tiene como objetivo, colaborar con la política energética nacional mediante la I+D+i y comercialización, centrándose en tres ejes fundamentales e innovadores que son 1) la implementación y optimización energética y ambiental, de procesos agroindustriales de cultivos bioenergéticos, 2) la producción de biocombustibles de tercera generación (producidos a partir de microrganismos), y, 3) soluciones bioenergéticas y mejoramiento de las propiedades de los biocombustibles mediante herramientas de nano y biotecnología.

Actualmente, LANOTEC trabaja en varios proyectos en asociación con dicha empresa, y, a su vez, la empresa trabaja en asociación con otras empresas, por ejemplo, la empresa pública estadounidense Alternative Fuels Americas Inc. (AFAI) y la empresa nacional Agronegocios de Costa Rica S.A.

Como iniciativa de colaboración con el VI Plan Nacional de Energía 2012-2030 de Costa Rica, así como con el Plan Nacional de Ciencia, Tecnología e Innovación 20112014, y el Programa Nacional de Biocombustibles, el laboratorio y la empresa, entes estatal y privado, se han asociado, tanto para colaborar con los planes de desarrollo anteriormente citados, como para empezar a agilizar y proponer los nuevos modelos de innovación con alianzas PYMES-academia implementados exitosamente en países desarrollados.

Hoy por hoy, LANOTEC y BSCA desarrollan, entre otras iniciativas, proyectos consistentes en el diseño e implementación de una planta piloto de extracción de aceites de microrganismos oleaginosos; un método alternativo altamente eficiente para la transesterificación de dichos aceites; así como el mejoramiento de las propiedades de biocombustibles en general, todo utilizando herramientas de la nanotecnología. El objetivo máximo de dichos proyectos es el escalamiento industrial de estos procesos y poner en el mercado nacional un biodiesel de alta calidad, que contribuya a cubrir la creciente demanda energética nacional.

Parte de estos proyectos son financiados, además de LANOTEC y la empresa, por la Alianza en Energía y Ambiente con Centroamérica (AEA).

Se está en la elaboración de más iniciativas de este tipo, bajo la alianza LANOTECPYMES, para el impulso de la I+D+i en materia energética, con miras a solventar los problemas energéticos nacionales, con el consecuente desarrollo nuevas empresas de alta tecnología que impulsen un encadenamiento económico que impacte de manera importante el desarrollo del país. 


\section{Referencias}

Ministerio de Ambiente y Energía. Ministerio de Agricultura y Ganadería. (2008). Programa Nacional de Biocombustibles. MAG-MINAE. República de Costa Rica.

Ministerio de Ambiente, Energía y Telecomunicaciones. Dirección Sectorial de Energía. (2011). VI Plan Nacional de Energía 2012-2030. MINAET-DSE. República de Costa Rica.

Ministerio de Ciencia y Tecnología. (2011). Plan Nacional de Ciencia, Tecnología e Innovación 2011-2014. ISBN: 978-9968-732-33-8. República de Costa Rica. 